\title{
Convalescent plasma associates with reduced mortality and improved clinical trajectory in patients hospitalized with COVID-19
}

\author{
Shanna A. Arnold Egloff, ${ }^{1,2}$ Angela Junglen, ${ }^{1,2,3}$ Joseph S.A. Restivo, ${ }^{2}$ Marjorie Wongskhaluang, ${ }^{2}$ Casey Martin, ${ }^{1,2,3}$ Pratik Doshi, ${ }^{1,2,3}$ \\ Daniel Schlauch, ${ }^{1,3}$ Gregg Fromell, ${ }^{1,2}$ Lindsay E. Sears, ${ }^{1,2}$ Mick Correll, ${ }^{1,3}$ Howard A. Burris III,, ${ }^{1,2}$ and Charles F. LeMaistre ${ }^{1,2}$ \\ 'Sarah Cannon, Nashville, Tennessee, USA. ${ }^{2}$ HCA Healthcare, HCA Research Institute (HRI), Nashville, Tennessee, USA. ${ }^{3}$ Genospace, Boston, Massachusetts, USA.
}

\begin{abstract}
BACKGROUND. Evidence supporting convalescent plasma (CP), one of the first investigational treatments for coronavirus disease 2019 (COVID-19), has been inconclusive, leading to conflicting recommendations. The primary objective was to perform a comparative effectiveness study of CP for all-cause, in-hospital mortality in patients with COVID-19.
\end{abstract}

METHODS. The multicenter, electronic health records-based, retrospective study included 44,770 patients hospitalized with COVID-19 in one of 176 HCA Healthcare-affiliated community hospitals. Coarsened exact matching (1:k) was employed, resulting in a sample of $3774 \mathrm{CP}$ and 10,687 comparison patients.

RESULTS. Examination of mortality using a shared frailty model, controlling for concomitant medications, date of admission, and days from admission to transfusion, demonstrated a significant association of CP with lower mortality risk relative to the comparison group (adjusted hazard ratio $[\mathrm{aHR}]=0.71 ; 95 \% \mathrm{Cl}, 0.59-0.86 ; P<0.001$ ). Examination of patient risk trajectories, represented by $\mathbf{4 0 0}$ clinico-demographic features from our real-time risk model (RTRM), indicated that patients who received $\mathrm{CP}$ recovered more quickly. The stratification of days to transfusion revealed that $\mathrm{CP}$ within 3 days after admission, but not within 4 to 7 days, was associated with a significantly lower mortality risk (aHR $=0.53 ; 95 \% \mathrm{Cl}, 0.47-0.60 ; P<0.001$ ). CP serology level was inversely associated with mortality when controlling for its interaction with days to transfusion (HR = $0.998 ; 95 \% \mathrm{Cl}, 0.997-0.999 ; P=0.013$ ), yet it did not reach univariable significance.

CONCLUSIONS. This large, diverse, multicenter cohort study demonstrated that CP, compared with matched controls, is significantly associated with reduced risk of in-hospital mortality. These observations highlight the utility of real-world evidence and suggest the need for further evaluation prior to abandoning CP as a viable therapy for COVID-19.

FUNDING. This research was supported in whole by HCA Healthcare and/or an HCA Healthcare-affiliated entity, including Sarah Cannon and Genospace.

\section{Introduction}

Convalescent plasma (CP) from patients who recover from severe acute respiratory syndrome coronavirus 2 (SARS-CoV-2) has been in use since the inception of the pandemic to treat coronavirus disease 2019 (COVID-19) (1-15). Discrepant reports of the efficacy of treatments for COVID-19, including CP, emerged from the rapidly evolving political and interventional landscape of the pandemic. Furthermore, clinical interpretations of this discordant data led to underuse, overuse, and misuse of certain interventions, often not considering the appropriate mechanistic context. Nevertheless, CP

Authorship note: SAAE and AJ contributed equally to this work and are co-first authors.

Conflict of interest: This research was supported by HCA Healthcare and/or an HCA Healthcare-affiliated entity. HAB reported grant funding from AstraZeneca, Medlmmune, Boehringer Ingelheim, Merck, Moderna Therapeutics, Verastem, Harpoon Therapeutics, Jounce Therapeutics, Janssen, BIND Therapeutics, Pfizer, Vertex, Gilead Sciences, Bayer, Incyte, Novartis, Seattle Genetics, GlaxoSmithKline, BioAtla, Agios, BioMed Valley Discoveries, TG Therapeutics, eFFECTOR Therapeutics, CicloMed, Array BioPharma, Roche/ Genentech, Arvinas, Bristol-Myers Squibb, Macrogenics, CytomX Therapeutics, Arch, Revolution Medicines, Lilly, Tesaro, Takeda/Millennium, Kyocera, Medlmmune, BIND Therapeutics, Kymab, miRNA Therapeutics, EMD Serono, and Foundation Medicine. HAB received consulting fees from Incyte, AstraZeneca, Celgene, and Forma Therapeutics. HAB consulted for Novartis, Bayer, Pfizer, GRAIL, Vincerx, and Daiichi Sankyo; received compensation for expert testimony from Novartis; and has stock ownership in HCA Healthcare. DS, MC, and SAAE report stock ownership in HCA Healthcare.

Role of funding source: The project was sponsored by HCA Healthcare at which all authors are employed directly or by an affiliate, Sarah Cannon and/or Genospace. HCA Healthcare prioritized and provided resources for this research and was involved in the decision to submit for publication. Data collection, analysis, and the writing of the report were performed independently by employees.

Submitted: May 28, 2021; Accepted: August 26, 2021; Published: October 15, 2021

Copyright: @ 2021, American Society for Clinical Investigation.

Reference information: J Clin Invest. 2021;131(20):e151788. https://doi.org/10.1172/JCI151788. 
has been utilized in prior pandemics and epidemics to introduce antibodies to elicit an immune response during the viral phase of infection. As a rapid response to an absence of any FDA-approved treatments of this deadly disease, CP use was granted through individual emergency investigational new drug (eIND) and the expanded access protocol (EAP) in the United States, providing sufficient safety data to justify emergency use authorization (EUA) in August of 2020 (16). Although over 100,000 patients were infused under the EAP, the absence of a matched comparison cohort limits the use of these data to assess the effectiveness of $\mathrm{CP}(8,17)$. Furthermore, randomized control trials (RCTs) struggled with enrollment, design, and cohort selection, nimbleness, and aligning site selection with local outbreaks, which resulted in underpowered and/or inconsistent conclusions on the efficacy of CP $(6,16,18-24)$. Most matched studies and RCTs around the globe have shown a trend of CP providing survival benefit, but all had relatively small cohorts except the RECOVERY trial $(n=11,558)$, which failed to show benefit with CP $(20,25)$. Two recent studies have demonstrated significantly reduced risk of hospital mortality in the CP-treated group, including a retrospective evaluation of hematologic cancer patients ( $n=966)$ and an RCT of severe/critical patients $(n=223)$, with $48 \%$ and $56 \%$ reduced risk, respectively $(26,27)$. Sluggish global deployment of vaccinations, diminished vaccine adoption rates, and the potential appearance of more transmissible and/or resistant variants have renewed interest in CP. Recent data from the EAP cohort revealed that regional proximity of donor $\mathrm{CP}$ to the recipient is associated with reduced mortality, suggesting that regional variations in SARS-CoV-2 could be driving CP responses (28).

Due to the limitations of RCTs in assessing CP during a rapidly evolving pandemic, well-matched, retrospective analyses are critical for comparative effectiveness studies, where they also serve to inform on utilization trends and generate hypotheses. Challenges for retrospective analyses to date have been the difficulty in accurately generating a matched synthetic control as well as having a sensitive indicator of disease progression and therapeutic response. We previously reported a real-time risk model (RTRM) for COVID-19 that provides a daily granular measure of disease progression to adequately match baseline disease severity and create a risk trajectory for each patient (29). Using both the daily RTRM probabilities and COVID-19 WHO progression scale (WHO PS), we retrospectively examined the association of $\mathrm{CP}$ with all-cause, in-hospital mortality and clinical recovery in matched cohorts derived from our COVID-19 registry. The registry consisted of 44,770 patients admitted to one of the $176 \mathrm{HCA}$ Healthcare-affiliated community hospitals where CP was provided under an eIND, EAP, or EUA.

The aim of the current study was to provide value to the growing body of literature surrounding CP benefit by providing insights from a large, diverse, and community-based COVID-19 cohort. To our knowledge, this is the largest retrospective study to date to evaluate the effectiveness of $\mathrm{CP}$ at reducing all-cause mortality in the overall hospitalized COVID-19 population by comparing effects with those of a comparison group matched to greater than 400 clinico-demographic features. Our data provide context for optimal delivery and validate recent trends in the literature showing CP benefit (25-27). Finally, there is a dose-response effect with $\mathrm{CP}$ antibody levels and we demonstrate that sooner CP adminis- tration really is better in accordance with the mechanisms of viral clearance and immune regulation.

\section{Results}

Patient characteristics. The WHO PS-matched sample resulted in $4337 \mathrm{CP}$ and 8708 comparison patients, and the RTRM-matched sample resulted in $3774 \mathrm{CP}$ and 10,687 comparison patients (Figure 1, Supplemental Tables 1-3 [WHO PS], and Tables 1, 2, 3 [RTRM]; supplemental material available online with this article; https://doi. org/10.1172/JCI151788DS1). The majority of the RTRM-matched sample subjects were Hispanic (49\%) or non-Hispanic White (32\%), male (60\%), and in the 45 - to 64-year age group (46\%) with predominant comorbidities of diabetes (30\%) and hypertension (49\%; Table 1). For both the CP and comparison groups, $12 \%$ of patients presented with severe sepsis and $3 \%$ with bacterial pneumonia during their hospitalization (Table 2). Although the difference was minimized by matching, the CP group retained higher rates of sepsis (32\%) compared with the comparison group (24\%; Table 2). After excluding those intubated within 2 days of admission and unlikely to receive CP in time to benefit, there were $1.9 \%$ of patients intubated at some point during hospitalization (Table 2). Most patients received anticoagulants, azithromycin, other antibiotics, remdesivir,and steroids during hospitalization (Table 2). Biomarker and oxygenation data were descriptively reported for both admission and baseline time points for both CP and comparison groups, which included standard unit transformation and validation of expected values (Table 3 ).

Details on descriptives and frequencies for the overall eligible cohort by calendar epoch can be found in Supplemental Tables 4 and 5. From the overall eligible cohort $(n=33,987)$ shown in Figure 1 , there were 8034 patients treated with CP and 25,953 patients not treated with CP. The mean number of days from admission to transfusion and length of stay across all epochs for the eligible cohort was $4.0 \pm 3.7$ and $14.9 \pm 10.9$, respectively (Supplemental Table 5). Distribution of the frequency of CP transfusions by calendar date was graphed, and the index time frame for active EAP was highlighted in order to estimate EAP enrollment compared with eIND and EUA, noting that eIND usage throughout the active EAP time frame could not be excluded based on structured health records (Supplemental Figure 1, 90\% EAP). In addition, we evaluated the incidence of transfusion-related serious adverse events, including transfusion-related acute lung injury (TRALI), transfusion-associated circulatory overload (TACO), transfusion-related infection, thromboembolic/thrombotic event, severe allergic transfusion reaction, severe hemolytic transfusion reaction, transfusion-related severe anaphylaxis, and unspecified transfusionrelated adverse events in patients who received CP (all CP; $n=$ 8034), fresh-frozen plasma verified negative for SARS-CoV-2 total antibody (verified FFP; $n=572$ ), or neither CP nor FFP with other blood products not ruled out (non-CP, non-FFP; $n=25,953$; Supplemental Table 6). A very low rate of transfusion-related adverse events was identified for all 3 cohorts. A total of 19 events $(0.23 \%)$ were identified for the eligible CP cohort as compared with $0.87 \%$ in the FFP and $0.11 \%$ in the non-CP, non-FFP cohorts (Supplemental Table 6). Differences in adverse events between $\mathrm{CP}$ and non-CP, non-FFP cohorts was predominantly due to TRALI and unspecified transfusion-related adverse events. Importantly, the 


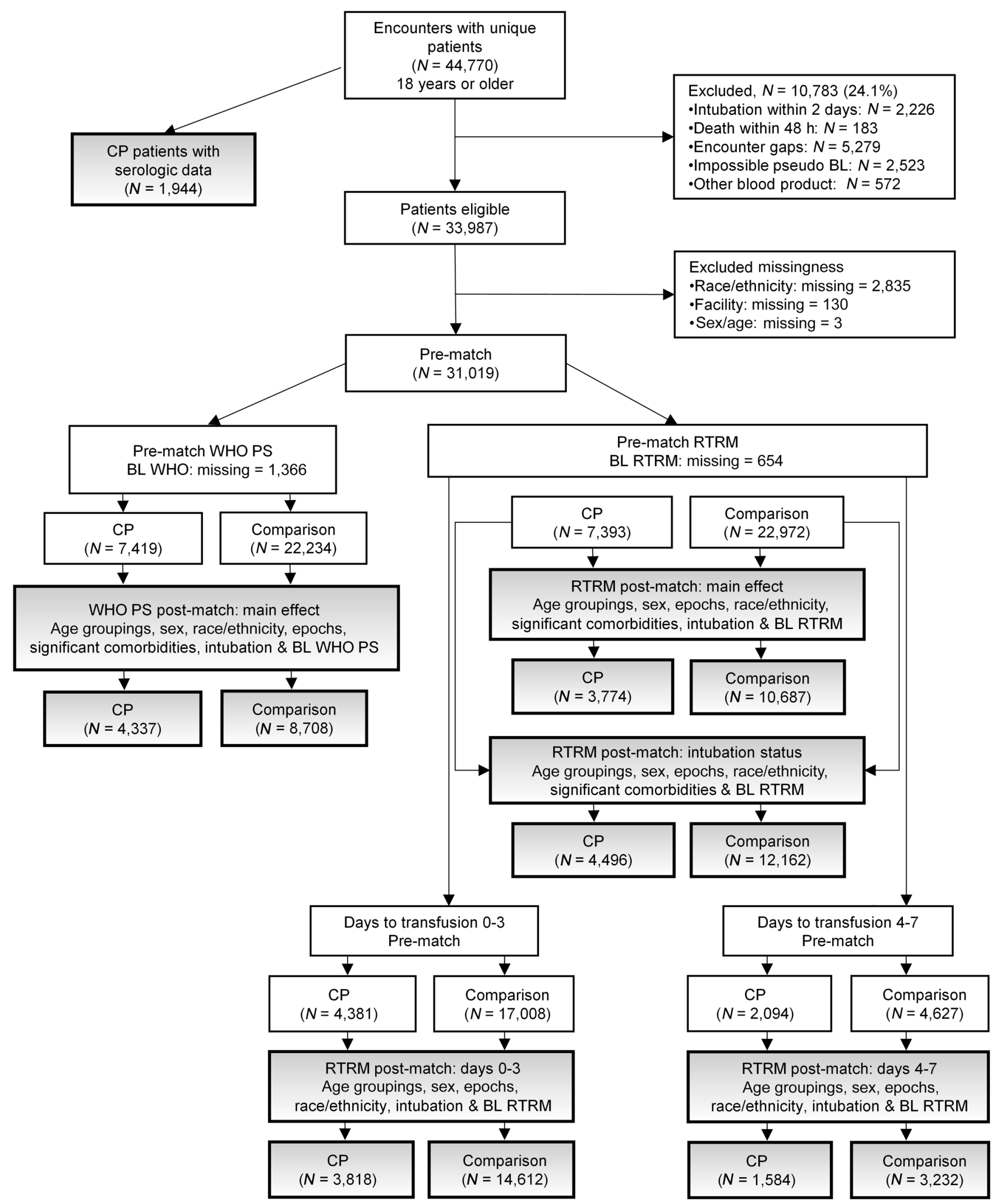

Figure 1. Patient selection and matching approaches. Consort diagram displaying patient inclusion/exclusion and filtering criteria for defining the COVID-19 cohort of interest for each analysis. Level of absent data is represented prior to coarsened exact matching (CEM). Subsequent prematch and postmatch patient counts are reported with relevant matching criteria that were applied. Gray boxes indicate final postmatch cohorts for all reported analyses (WHO PS: main effect; RTRM: main effect; RTRM: intubation status; RTRM: days to transfusion 0-3; RTRM: days to transfusion 4-7; and serologic data). Excluding patients intubated within 48 hours after admission, intubation status was defined as intubated at any time during hospitalization for matching purposes and intubated prior to or on day of transfusion for the intubation subgroup analysis. 


\section{Table 1. Demographics for RTRM-matched model}

\begin{tabular}{|c|c|c|c|c|}
\hline & Prematch CP & Prematch comparison & Postmatch CP & Postmatch comparison \\
\hline Total number of patients, $n$ & 7393 & 22972 & 3774 & 10687 \\
\hline \multicolumn{5}{|l|}{ Age groupings ${ }^{A}$} \\
\hline $18-44$ & $958(13.3 \%)$ & $3970(17.3 \%)$ & $713(18.9 \%)$ & $2019.03(18.9 \%)$ \\
\hline $65-74$ & $1707(23.1 \%)$ & $4516(19.7 \%)$ & $667(17.7 \%)$ & $1888.77(17.7 \%)$ \\
\hline $75-84$ & $1315(17.8 \%)$ & $4087(17.8 \%)$ & $456(12.1 \%)$ & $1291.28(12.1 \%)$ \\
\hline Hispanic & $3253(44.0 \%)$ & $7500(32.6 \%)$ & $1859(49.3 \%)$ & $5264.21(49.3 \%)$ \\
\hline Non-Hispanic, Black & $1150(15.6 \%)$ & $5177(22.5 \%)$ & $512(13.6 \%)$ & $1449.85(13.6 \%)$ \\
\hline Non-Hispanic, other ${ }^{B}$ & $460(6.2 \%)$ & $1309(5.7 \%)$ & $215(5.7 \%)$ & $608.82(5.7 \%)$ \\
\hline Non-Hispanic, White & $2530(34.2 \%)$ & $8986(39.1 \%)$ & $1188(31.5 \%)$ & $3364.11(31.5 \%)$ \\
\hline \multicolumn{5}{|l|}{$\operatorname{Sex}^{A}$} \\
\hline Former smoker & $1444(19.5 \%)$ & $3909(17.0 \%)$ & $609(16.1 \%)$ & $1623.89(15.2 \%)$ \\
\hline Never smoker & $4815(65.1 \%)$ & $13528(58.9 \%)$ & $2701(71.6 \%)$ & $6838.05(64.0 \%)$ \\
\hline ND & $871(11.8 \%)$ & $4213(18.3 \%)$ & $343(9.1 \%)$ & $1581.24(14.8 \%)$ \\
\hline \multicolumn{5}{|l|}{ Preadmission comorbidities } \\
\hline Asthma or reactive airway disease & $706(9.5 \%)$ & $2315(10.1 \%)$ & $282(7.5 \%)$ & $747.71(7.0 \%)$ \\
\hline COPD (excluding asthma) ${ }^{A}$ & $1424(19.3 \%)$ & $4769(20.8 \%)$ & $352(9.3 \%)$ & $996.77(9.3 \%)$ \\
\hline Autoimmune disorders & $879(11.9 \%)$ & $2826(12.3 \%)$ & $126(3.3 \%)$ & $356.80(3.3 \%)$ \\
\hline Organ-related autoimmune disorders ${ }^{A}$ & $436(5.9 \%)$ & $1789(7.8 \%)$ & $60(1.6 \%)$ & $169.90(1.6 \%)$ \\
\hline Systemic autoimmune disorders ${ }^{A}$ & $505(6.8 \%)$ & $1295(5.6 \%)$ & $69(1.8 \%)$ & $195.39(1.8 \%)$ \\
\hline Cancer & $421(5.7 \%)$ & $1442(6.3 \%)$ & $48(1.3 \%)$ & $135.92(1.3 \%)$ \\
\hline Cancer (diagnosed in the last 2 years) & $228(3.1 \%)$ & $795(3.5 \%)$ & $28(0.7 \%)$ & $71.27(0.7 \%)$ \\
\hline Renal disease (mild or moderate) ${ }^{A}$ & $1147(15.5 \%)$ & $3943(17.2 \%)$ & $298(7.9 \%)$ & $843.86(7.9 \%)$ \\
\hline Renal disease (severe) & $491(6.6 \%)$ & $1375(6.0 \%)$ & $65(1.7 \%)$ & $184.06(1.7 \%)$ \\
\hline
\end{tabular}

Categorical data are shown as $n(\%)$, and continuous variables are presented as median (IQR). ${ }^{A}$ Variables used for CEM matching. ${ }^{8}$ Other is defined as $60 \%$ recorded in the medical record as "other" race where the remaining $40 \%$ includes reported race of American Indian/Alaska Native, Asian, Asian Indian, Native Hawaiian/other Pacific Islander, or multiracial. COPD, chronic obstructive pulmonary disease.

CP cohort did not have greater rates of adverse events compared with the FFP-transfused cohort, suggesting that CP transfusion posed no higher risk than other transfusions.

All-cause mortality. For main analyses examining the effect of CP on all-cause, in-hospital mortality, both the WHO PS-matched and the RTRM-matched models included matching variables of calendar epoch, intubation any time during hospitalization (excluding those intubated within 48 hours of admission), age grouping, ethnicity/race, sex, significantly different prematch comorbidities, and severity, measured by the WHO PS score or the RTRM risk probability (using 0.10 increments) at baseline. Significant comorbidities included organ-specific autoimmune disorder, systemic autoimmune disorder, chronic pulmonary disease (excluding asthma), diabetes (without complications), mild/moderate renal disease, cancer, congestive heart failure, and chronic ischemic heart disease (Table 1 and Supplemental Table 1).

When examining all-cause, in-hospital mortality using a shared frailty model to account for facility effects, both models, WHO PS matched and RTRM matched, demonstrated a significant association of CP with lower risk of mortality compared with the matched comparison group (WHO PS: adjusted hazard ratio [aHR] $=0.75 ; 95 \%$ CI, 0.65-0.85; $P<0.001$; RTRM: $\mathrm{aHR}=0.71$; 95\% CI, 0.59-0.86; $P<0.001)$ when controlling for concomitant medications, calendar date of admission, and days from admission to transfusion (Table 4). Coinfections such as bacterial pneumonia, sepsis, and severe sepsis were only controlled for 
Table 2. Clinical characteristics for RTRM-matched model

\begin{tabular}{|c|c|c|c|c|}
\hline & Prematch CP & Prematch comparison & Postmatch CP & Postmatch comparison \\
\hline Total number of patients, $n$ & 7393 & 22972 & 3774 & 10687 \\
\hline \multicolumn{5}{|l|}{ Medications } \\
\hline Anticoagulants & $7232(97.8 \%)$ & $19647(85.5 \%)$ & $3688(97.7 \%)$ & $9519.65(89.1 \%)$ \\
\hline Azithromycin & $5447(73.7 \%)$ & $14123(61.5 \%)$ & $2767(73.3 \%)$ & $6991.49(65.4 \%)$ \\
\hline Antibiotics, other & $6711(90.8 \%)$ & $18641(81.1 \%)$ & 3278 (86.9\%) & $8761.25(82.0 \%)$ \\
\hline Antivirals & $164(2.2 \%)$ & $474(2.1 \%)$ & $54(1.4 \%)$ & $160.58(1.5 \%)$ \\
\hline Hydroxychloroquine & $429(5.8 \%)$ & $2580(11.2 \%)$ & $162(4.3 \%)$ & $331.29(3.1 \%)$ \\
\hline Remdesivir & $4549(61.5 \%)$ & $3162(13.8 \%)$ & $2240(59.4 \%)$ & $2206.96(20.7 \%)$ \\
\hline Tocilizumab & $784(10.6 \%)$ & $554(2.4 \%)$ & $251(6.7 \%)$ & $353.30(3.3 \%)$ \\
\hline Statins/ACEi & 2962 (40.1\%) & $8322(36.2 \%)$ & $1209(32.0 \%)$ & $3156.30(29.5 \%)$ \\
\hline Systemic corticosteroids & $6994(94.6 \%)$ & $12988(56.5 \%)$ & $3563(94.4 \%)$ & $7453.14(69.7 \%)$ \\
\hline Immunomodulators, other & $11(0.15 \%)$ & $22(0.1 \%)$ & $3(0.1 \%)$ & $5.92(0.1 \%)$ \\
\hline \multicolumn{5}{|l|}{ Clinical variables } \\
\hline Intubation ${ }^{A}$ & $1290(17.4 \%)$ & $815(3.5 \%)$ & $71(1.9 \%)$ & $201.05(1.9 \%)$ \\
\hline All-cause death & $1519(20.5 \%)$ & $1500(6.5 \%)$ & $219(5.8 \%)$ & $477.90(4.5 \%)$ \\
\hline Baseline RTRM score $^{A}$ & $0.14(0.04,0.45)$ & $0.04(0.02,0.13)$ & $0.05(0.02,0.12)$ & $0.03(0.01,0.12)$ \\
\hline \multicolumn{5}{|l|}{ Admission WHO PS } \\
\hline WHO PS 2 & $869(11.8 \%)$ & 8019 (34.9\%) & $463(12.3 \%)$ & $3427.69(32.1 \%)$ \\
\hline WHO PS 3 & $3816(51.6 \%)$ & $11382(49.5 \%)$ & $2255(59.8 \%)$ & $5316.54(49.7 \%)$ \\
\hline WHO PS 4 & $2524(34.1 \%)$ & $2262(9.8 \%)$ & $1013(26.8 \%)$ & $1365.30(12.8 \%)$ \\
\hline WHO PS 5 & $149(2.0 \%)$ & $283(1.2 \%)$ & $29(0.8 \%)$ & $149.66(1.4 \%)$ \\
\hline ND & $35(0.5 \%)$ & $1026(4.5 \%)$ & $14(0.4 \%)$ & $427.81(4.0 \%)$ \\
\hline \multicolumn{5}{|l|}{ Baseline WHO PS } \\
\hline WHOPS 2 & $247(3.3 \%)$ & 7335 (31.9\%) & $175(4.6 \%)$ & $3072.24(28.7 \%)$ \\
\hline WHO PS 3 & $2503(33.8 \%)$ & 11312 (49.2\%) & $1827(48.4 \%)$ & $5238.54(49.0 \%)$ \\
\hline WHO PS 4 & 3994 (54.0\%) & $2898(12.6 \%)$ & 1701 (45.1\%) & $1801.22(16.9 \%)$ \\
\hline WHO PS 5 & $637(8.6 \%)$ & $536(2.3 \%)$ & $61(1.6 \%)$ & $207.07(1.9 \%)$ \\
\hline ND & $12(0.2 \%)$ & $891(3.9 \%)$ & $10(0.3 \%)$ & $367.93(3.4 \%)$ \\
\hline \multicolumn{5}{|l|}{ Secondary infections } \\
\hline Bacteremia & $44(0.6 \%)$ & $155(0.6 \%)$ & $15(0.4 \%)$ & $66.80(0.6 \%)$ \\
\hline Bacterial pneumonia & $432(5.8 \%)$ & $682(3.0 \%)$ & $116(3.1 \%)$ & $271.09(2.5 \%)$ \\
\hline Sepsis & 2022 (37.4\%) & $5019(21.8 \%)$ & $1202(31.8 \%)$ & $2547.75(23.8 \%)$ \\
\hline Severe sepsis & $1878(25.4 \%)$ & $2681(11.7 \%)$ & $453(12.0 \%)$ & $1235.86(11.6 \%)$ \\
\hline
\end{tabular}

Categorical data are shown as $n$ (\%), and continuous variables are presented as median (IQR). Intubation for matching defined as yes/no intubation at any time during hospitalization after excluding those intubated within 48



treated with $\mathrm{CP}$ were associated with a quicker decline in risk severity over time than the matched comparison group for both overall hospitalization (CP, slope $[b]=-0.0038$; comparison, $b=-0.0030)$ and the first 10-day window (CP, $b=-0.0040$; comparison, $b=-0.0034)$, respectively. This -0.0008 difference in slope equated to an overall $27 \%$ difference in risk reduction per day of hospitalization for CP relative to the comparison group. The RTRM risk trajectory analyses assumed a linear fit, so we provided average daily RTRM probabilities over hospitalization to observe nonlinear trends in disease progression for the RTRM main effect, intubation status, and days to transfusion (Figure 2).

Intubation status subgroups. Intubation status was defined by whether patients were intubated prior to or on same day as transfusion or pseudo-baseline, but excluded those patients intubated within the first 48 hours after admission. Statistical analyses examined the interaction of $\mathrm{CP}$ with intubation, which served as a surrogate for baseline severity, in relation to all-cause, in-hospital mortality. The shared frailty analysis showed that the effect of $\mathrm{CP}$ on risk of mortality did not differ for patients intubated prior to or at baseline com-

in the WHO PS-matched model, since the RTRM accounts for secondary infections.

Both the WHO PS-matched model and RTRM-matched model violated the assumption of proportional hazards, so accelerated failure time (AFT) models were performed to examine the consistency of the effect (Table 2). The AFT did replicate the main findings of CP on life expectancy for both models, but the AFT models did not account for facility effects (WHO PS: adjusted deceleration factor $[\mathrm{aDF}]=1.21 ; 95 \% \mathrm{CI}, 1.06-1.38 ; P=0.005$; RTRM: aDF $=1.23$; $95 \% \mathrm{CI}, 1.10-1.37 ; P=0.005)$.

$R T R M$ risk trajectories. We investigated the effect of $\mathrm{CP}$ on rate of recovery using a mixed effects model with the RTRM-matched sample to evaluate RTRM trajectories over time and account for facility effects. CP was significantly associated with a quicker RTRM score improvement than in the matched comparison group, controlling for concomitant medications, calendar date of admission, and days from admission to transfusion $(P<0.001)$. Patients pared with nonintubated patients, as demonstrated by including an interaction term in the model and controlling for concomitant medications, calendar date, and days from admission to transfusion $(P=0.160$; Table 5).

Days from admission to transfusion. There was a significant effect of days from admission to transfusion on mortality, specifically within the CP group $(\mathrm{aHR}=1.06$; 95\% CI, 1.03-1.09; $P<$ 0.001). Therefore, exploratory analyses were performed to detail this effect. A Cox regression model was progressively run, splitting by every number of days from admission to transfusion, and it was noted that the upper $95 \% \mathrm{CI}$ of the aHR did not cross 1.0 out to 7 days. However, if each number of days was run in isolation, 3 days from admission was the limit due to decreasing sample size.

Therefore, we examined the effect of CP across days on mortality risk by stratifying the RTRM sample into 2 groups, 0 to 3 and 4 to 7 days, in accordance with sample distribution. Each CP group was matched to comparison on baseline RTRM 


\section{Table 3. Admission and baseline biomarkers for RTRM-matched model}

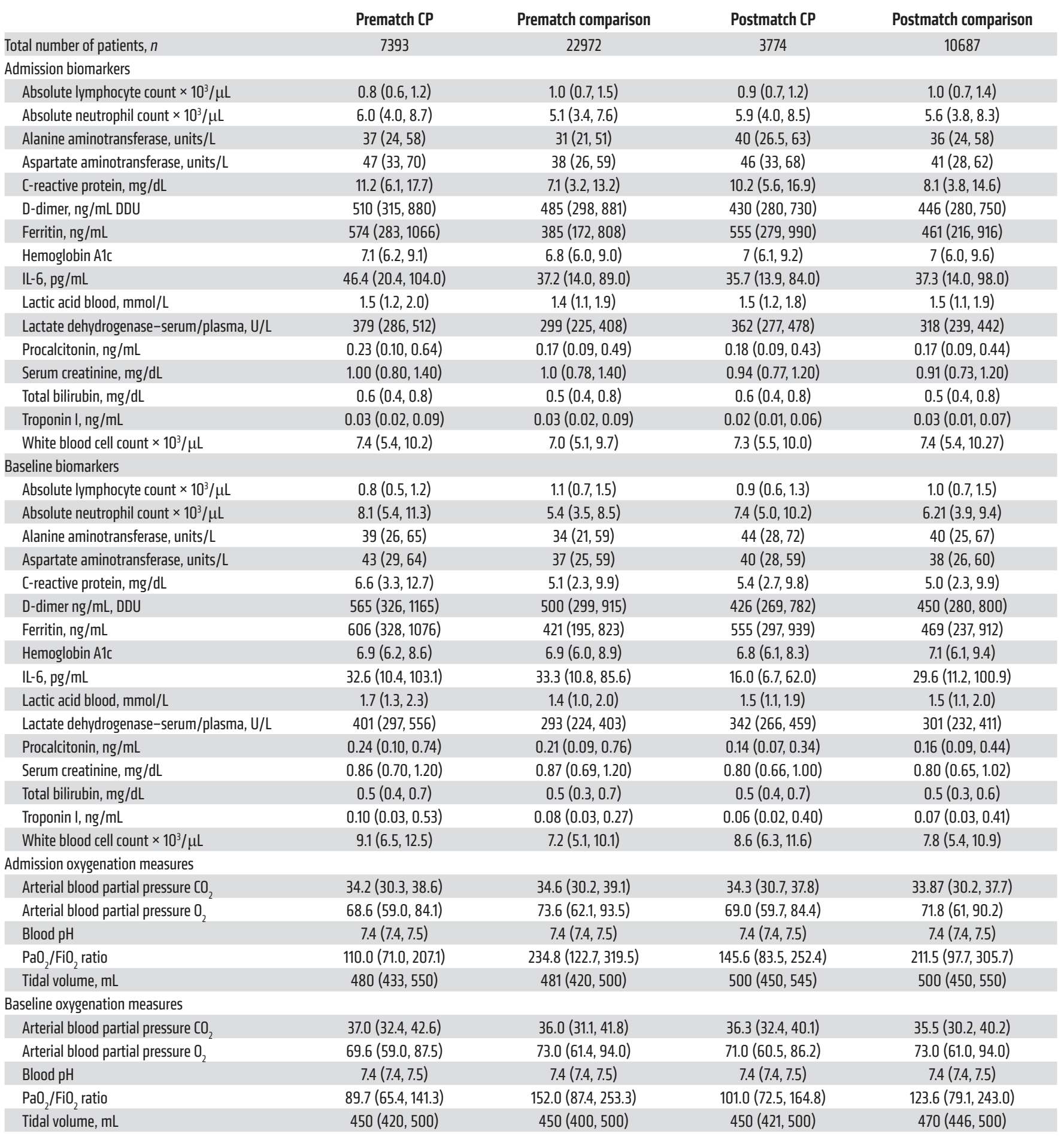

Categorical data are shown as $n(\%)$, and continuous variables are presented as median (IQR). DDU, D-dimer units.

probability, sex, age, and race. We identified a significant association of $\mathrm{CP}$ with reduced mortality risk when examining the 0 - to 3-day group (aHR $=0.53 ; 95 \% \mathrm{CI}, 0.47-0.60 ; P<0.001$ ), but this association was not significant for the 4- to 7-day group (aHR $=0.94 ; 95 \% \mathrm{CI}, 0.76-1.15 ; P=0.520)$ after adjusting for concomitant medications, calendar date, and days from admis- sion to transfusion, suggesting the significant association of days from admission to transfusion is driven by the first 3 days (Table 5). However, this should not be interpreted as showing there is no benefit after 3 days with CP, as the distribution of our sample was dominated by CP transfusions within 3 days after admission (66\%; Supplemental Figure 2). 
Table 4. Effects across multivariable all-cause hospital mortality models

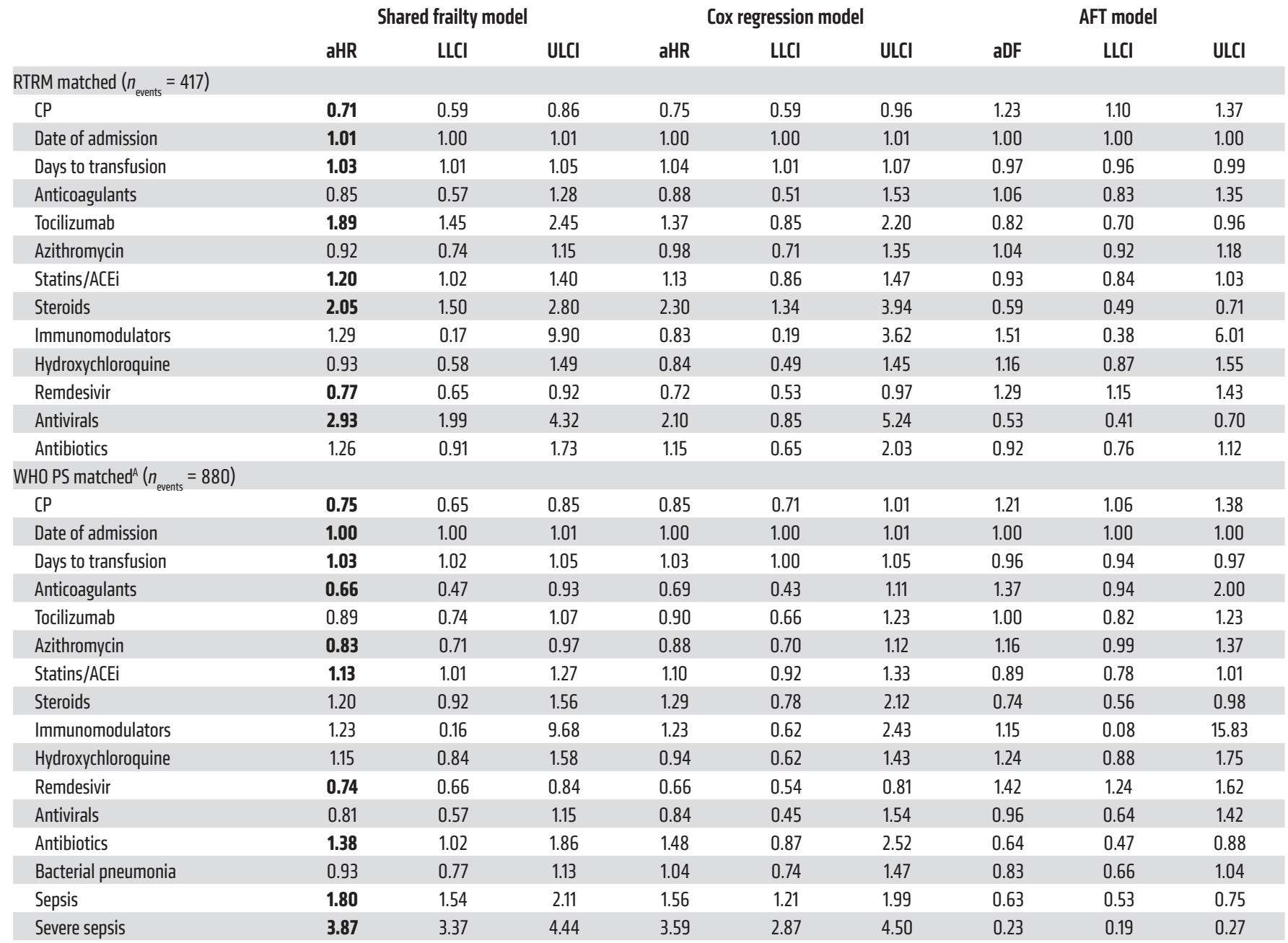

Medications were computed as indicators of usage over the length of hospitalization. Effects provided are from multivariable analyses. ${ }^{A} \mathrm{WHO}$ PS model included secondary infections since all other models were matched on RTRM score, which would have accounted for secondary infections. LLCI, lower level of $95 \% \mathrm{Cl}$; $\mathrm{ULCl}$, upper level of $95 \% \mathrm{Cl}$. Numbers in bold text indicate significance (Cl not crossing 1.0).

Donor serology. When examining total anti-SARS-CoV-2 signal to cutoff ratio (S/Co) serology for donor samples as either a continuous variable or as ordinal low, medium, or high (20th and 80th percentiles), there was no significant association with risk of mortality, although there was a trend in the expected direction ( $n=1,944$, Supplemental Table 7 and ref. 8). To further this exploration, we determined whether the impact of donor serologic levels on mortality was influenced by days from admission to transfusion. The interaction of S/Co serology level as a continuous variable with days to transfusion was significantly associated with risk of mortality $(P=0.044)$ along with the main effects (serology: $\mathrm{HR}=0.998 ; 95 \% \mathrm{CI}, 0.997-0.999 ; P=0.013$; days to transfusion: HR $=1.036 ; 95 \%$ CI, 1.002-1.071; $P=0.037$ ) (Supplemental Table 7). This resulted in a $0.2 \%$ decreased risk of mortality for every 1 unit of S/Co serology level, where it ranged from 1.25 to 932.00 with a mean of 178.21 and SD of 138.49. Simple slopes analyses were performed to examine this significant interaction and are reported in Supplemental Table 7.

\section{Discussion}

Although others have shown a correlation of the levels of antibody titers in CP with improved outcomes and there are clear trends toward benefit across the COVID-19 CP literature $(8,25)$, our data are the first to our knowledge to provide evidence in a nationwide, community-based matched cohort that $\mathrm{CP}$ is associated with a 29\% reduced risk of death in patients hospitalized with COVID-19. This effect is even more pronounced if CP is delivered within the first 3 days after admission, revealing a $47 \%$ reduction in risk of in-hospital mortality for all patients regardless of baseline severity. However, detailed analyses suggest there might be continued benefit beyond day 3, indicating further investigations are warranted. Moreover, patients treated with CP experienced a faster recovery, equating to a $27 \%$ difference in reduction in risk/severity per day over their length of stay, as measured by daily RTRM probabilities. Interestingly, this effect was not dependent on baseline severity, as we found no differential association of $\mathrm{CP}$ with mortality risk based on intubated status prior to or at baseline. 
A RTRM post-match: main effect

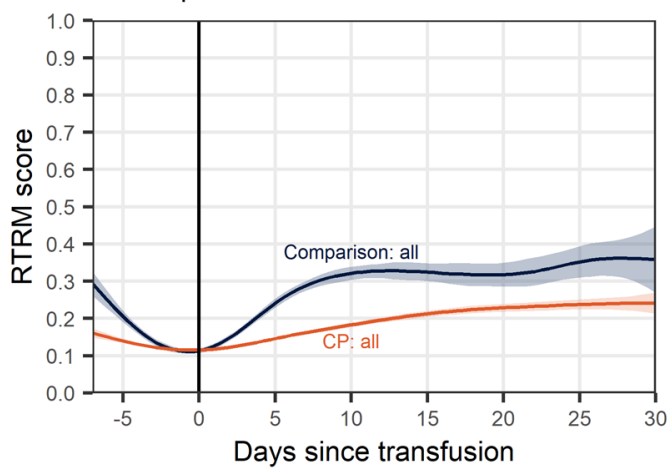

Days since transfusion

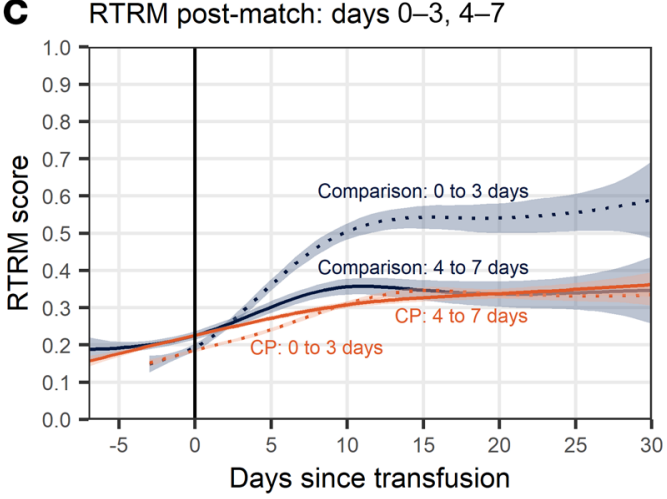

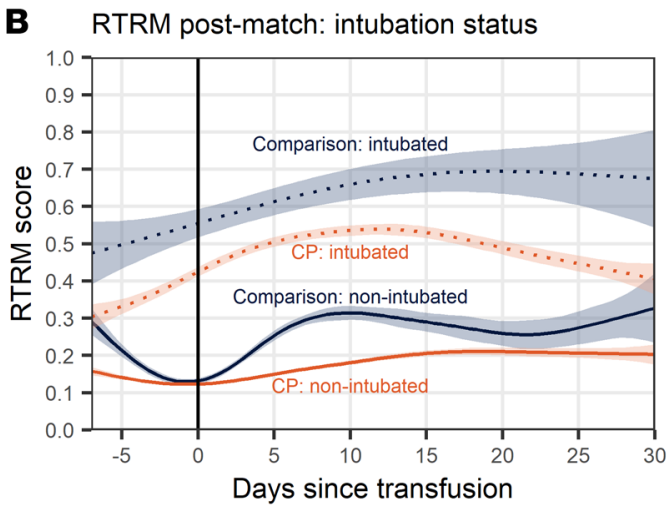

Days since transfusion

Figure 2. Real-time risk curves as a visual of daily patient risk trajectories. RTRM curves, smoothed and weighted, showing risk trajectories across CP and the comparison group. A generalized additive model with integrated smoothness estimation was applied to the risk predictions over hospitalization time, which were anchored by baseline date. Patients at discharge were assigned a final RTRM probability based on vital status of 1.00 for expired and 0.00 for alive. Shaded boundaries around each curve represent the $95 \% \mathrm{Cls}$. (A) Main weighted comparison of the RTRM-matched cohort of CP (orange) and the comparison (blue) groups. (B) Weighted comparison of CP (orange) and comparison (blue) groups stratified by intubation (dotted) versus no intubation (solid) at any point during hospitalization, excluding intubation within 48 hours after admission. Note, this is different from the intubation subgroup analysis where intubation was defined as having occurred prior to or on the day of transfusion or pseudo-baseline, excluding patients intubated within the first 48 hours of admission. (C) Compilation of weighted comparisons between CP (orange) and the comparison (blue) groups for each of the two 0 to 3 (dotted) and 4 to 7 (solid) days from admission to transfusion groupings.

Donor SARS-CoV-2 antibody levels for a subset of CP recipients, as measured by total $\mathrm{S} / \mathrm{Co}$, were associated with a $0.2 \%$ reduced risk of mortality per unit increase after adjusting for its interaction with days from admission to transfusion. Joyner et al. have also shown within the large EAP cohort that semiquantitative $\mathrm{CP}$ antibody levels were inversely correlated with mortality when given within a few days of hospitalization (8). Despite methodological differences in laboratory approaches and serology assay platforms used in the two studies, the consistency across these studies suggests there is, indeed, a dose-response benefit to receiving CP.

Although we made substantial efforts to manage challenges of analyzing real-world data, there are limitations inherent in their use. These include the evolution of diagnosis and treatment during this pandemic as well as changes in medical documentation and coding related to COVID-19 across multiple facilities. We attempted to account for this by matching on calendar epochs, controlling for calendar date, and nesting on facility. Neither BMI nor smoking status could be included in our analyses due to unreliability and absence of reported data, respectively. Furthermore, due to the retrospective, records-based design of this study combined with the community-based nature of our healthcare network, SARS-CoV-2-specific biomarkers such as viral load and recipient antibody levels before and after $\mathrm{CP}$ transfusion were not ordered as a standard of care and thus were not available in the medical records. Incorporating days from symptom onset to transfusion, rather than days from admission, could more accurately identify optimal timing of CP transfusion. However less than $10 \%$ of patients had clear reporting of symptom onset and thus we could not confidently execute analyses with symptom onset due to absence of data. Additionally, we defined intubations within the first 48 hours after admission as key exclusion criteria a priori due to the mechanistic assessment that $\mathrm{CP}$ would not have time to improve outcome of hospitalized patients admitted with the most severe disease symptomology generally due to overactive immune and chemokine responses. Looking at rates of patients excluded based on intubation within the first 48 hours from the CP cohort (9.6\%) as compared with the comparison group (5.1\%) revealed that there was a small selection bias. However, this bias did not affect our current analyses, since baseline severity for the prematch CP cohort remained higher than baseline severity for the prematch comparison group in regard to intubation status, RTRM probabilities, and WHO PS scores. Finally, although concomitant medications were controlled for in all primary, secondary, and subgroup analyses, they were treated as indicators during 
Table 5. Effects across multivariable mortality models for all subanalyses

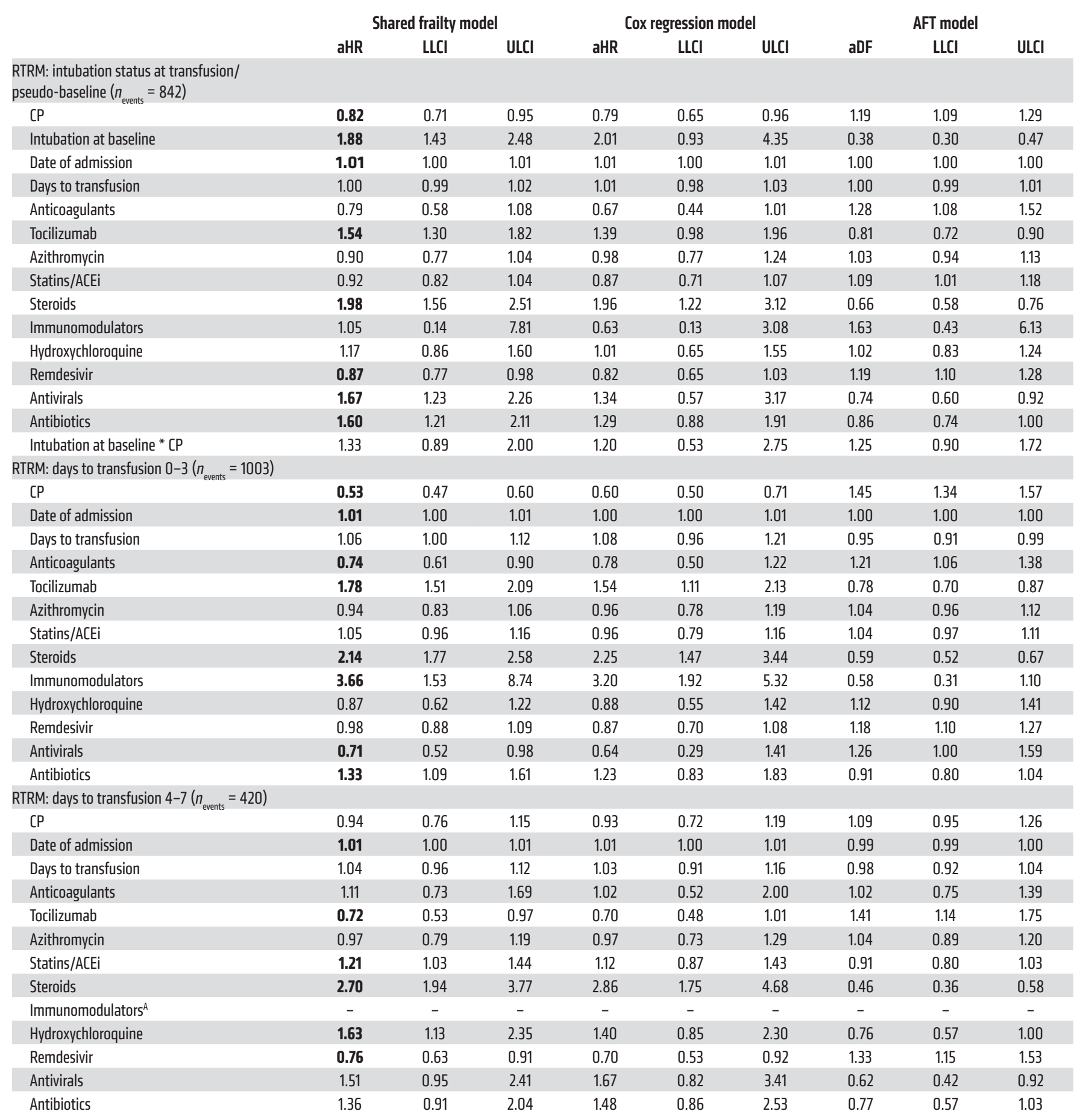

Medications were treated as indicators of usage over the length of hospitalization. Effects provided are from multivariable analyses. Intubation status at transfusion or pseudo-baseline for the subanalysis defined as intubated prior to or on day of baseline, excluding those intubated within 48 hours after admission. Almmunomodulators were removed from days to transfusion 4-7 model since there were minimal occurrences $(n=2)$. Numbers in bold text indicate significance ( $\mathrm{Cl}$ not crossing 1.0).

hospitalization rather than accounting for their timing with $\mathrm{CP}$ and dose. Future studies will be directed at evaluating medication interactions with CP and identifying optimal dosing and timing of concomitant medications.
A challenge to retrospectively evaluating CP delivered in the community setting has been the evolution of treatment criteria and access to CP. The CP cohort was skewed to higher severity based on eligibility criteria for EAP enrollment prior to approval 
for EUA. Inconsistencies in supply and demand occurred throughout 2020 in various outbreak locations. These challenges made it difficult for most research groups to create a well-matched comparison cohort; however, we were able to capitalize on the granularity of our RTRM and the coarsened exact matching approach to match on hundreds of clinico-demographic and biomarker features to identify a properly matched cohort (29). Although RCTs are the gold standard for assessing efficacy, their deployment during a rapidly evolving pandemic is especially challenging at scale and in the community setting. Our robust comparative effectiveness evaluation of CP utilizing the large and diverse HCA Healthcare COVID-19 registry for rigorous matching demonstrates a significant reduction in mortality in hospitalized patients, especially those treated within 3 days of admission $(\mathrm{aHR}=0.53$; $95 \% \mathrm{CI}, 0.47-0.60 ; P<0.001)$. This retrospective study comes as close to mitigating the biases arising from nonrandomized treatment assignment as it is possible to achieve. Indeed, our data align with two recently published studies, including a multisite RCT and a large retrospective study in hematologic malignancies, that demonstrate a $56 \%$ reduction in 28 -day and a $48 \%$ reduction in 30 -day in-hospital mortality, respectively $(26,27)$. Finally, these results corroborate work from Casadevall and colleagues showing an inverse correlation (-0.52) between the number of COVID-19 deaths occurring within 2 weeks of hospital admission and CP usage within the United States (30). We believe our data to be applicable to diverse groups as well as important in contemplating design of future RCTs.

As novel and more virulent and transmissible SARS-CoV-2 variants emerge around the globe and as reports of post-vaccine "breakthrough" infections and vaccine hesitancy increase, there is a renewed motivation to identify effective treatments for hospitalized patients. The data presented here demonstrate that further evaluation is required prior to abandoning $\mathrm{CP}$ as an effective intervention in the treatment of hospitalized COVID-19 patients.

\section{Methods}

\section{Data sources}

The design, analysis, and data interpretations were conducted independently by the investigators. All authors testify to the accuracy and completeness of the data with acknowledgement that there are limitations to real-world evidence. Data for the study were obtained through HCA Healthcare's data warehouse, which contains detailed and structured clinico-demographic, medical, medical history, pharmacy, laboratory, and outcomes data captured in electronic medical record systems (Epic, Cerner, Meditech) of 176 HCA Healthcareaffiliated clinics and community hospitals across the United States. Any data captured from March 2 to October 7, 2020, were included in the study, allowing for a minimum of 10-day follow-up, while still retaining $99 \%$ of the initial sample with available discharge dates (Figure 1).

\section{Patient selection}

Patients 18 years or older that were hospitalized at any of HCA Healthcare's hospitals and tested positive (CDC confirmed) or presumptive positive (not CDC confirmed) for SARS-CoV-2 by any assay platform (PCR, rapid antigen, antibody, etc.) 4 weeks before to 2 weeks after their admission date were eligible for the study. Patients were excluded if they were intubated or expired within the first 48 hours after admission as defined a priori. Any data from prior or subsequent admissions or transfers within 36 hours were linked to create a continuum of patient care (Supplemental Methods). Patients were excluded if there were discordant gaps in care across data sources (Figure 1).

\section{Exposure}

The primary exposure for comparison was the first transfusion of $\mathrm{CP}$ for treatment of COVID-19. We identified CP exposure by patients having a transfusion date and receiving a blood product with a variation of convalescent and/or COVID in the name and a corresponding ISBT-128 CP barcode as confirmed by blood-bank suppliers. Patients who received unconfirmed plasma products were excluded from analyses. Patients were included regardless of whether CP access was provided under an eIND, EAP, or EUA. Patients who did not receive transfusion of $\mathrm{CP}$ or any other plasma product were considered as the comparison group.

We were able to obtain donor serology data measuring total antiSARS-CoV-2 antibodies from blood-bank suppliers used to treat a subset of hospitalized COVID-19 patients. At the time, these suppliers were only able to provide the donor serology results for the VITROS SARSCoV-2 Total Antibody Test (CoV2T, Ortho Clinical Diagnostics), which targets the $\mathrm{S} 1$ antigen, detects IgM, IgA, and IgG antibodies, has a large linear range, and has a sensitivity and specificity of $90 \%$ and $100 \%$, respectively (16). The American Red Cross correlated CoV2T S/Co to neutralizing titers using plaque reduction neutralization assay (PRNT) and showed a linear correlation between CoV2T and PRNT, with $82 \%$ of samples with 1 or more total S/Co having a high neutralizing titer of 1:160 or more (31-33). Therefore, positive serology was defined as a S/Co greater than $1(n=1944)$, where all positives were evaluated for correlation with outcome. Mean donor serology levels were calculated for patients receiving multiple units of $\mathrm{CP}$, whether concurrently or in subsequent transfusions within a week of the first CP transfusion. Due to low sample size in comparison with the HCA COVID-19 registry cohort, donor serology levels were not included as a covariate. Exploratory analyses were performed as described in Results.

The day of the first CP transfusion was considered baseline (day 0) for the CP group. The comparison group was randomly assigned a pseudo-baseline prior to matching that reflected distribution of the time interval from admission to transfusion equal to that of the $\mathrm{CP}$ group (Supplemental Methods).

\section{Outcomes measures}

All-cause in-hospital mortality (primary outcome). All patients were required to have a discharge date to be included in analyses. Patient vital status at time of discharge delineated censored from expired. Start time for all analyses was defined by baseline, which was the first CP transfusion date for the CP group and the assigned pseudo-baseline for the comparison group (Supplemental Methods).

RTRM risk trajectory (secondary outcome). Daily mortality risk scores were generated for all patients across the length of their hospitalization using probabilities from our COVID-19 RTRM, which incorporates hundreds of structured medical record features, such as clinico-demographics, comorbidities, laboratory values, secondary infections, complications, oxygenation details, and oxygen supplementation (29). When using the daily RTRM probabilities as a longitudinal outcome measure of progression and/or recovery, we also 
included baseline RTRM probability as a matching variable. Medications were purposely excluded from the RTRM model to be evaluated as covariates in comparative effectiveness studies such as this (Supplemental Table 8).

\section{Data sharing}

The data that support the findings of this study are available upon request from the corresponding author. The data are not publicly available due to privacy restrictions. A limited data set could be made available under a transfer agreement.

\section{Statistics}

Matching. All baseline patient characteristics were compared between the CP and comparison groups using 2-tailed $t$ tests for continuous variables and $\chi^{2}$ tests for categorical variables. To mitigate bias resulting from nonrandomized assignment of treatment, a coarsened exact matching technique was used to match patients in the comparison group to the CP group (34). The matching for the main analyses was done based on patient age groupings, sex, race/ethnicity, significantly different comorbidities, calendar epoch, intubation status at any time during hospitalization (excluding those intubated within 48 hours of admission), and baseline severity (WHO PS or RTRM probability in 0.10 increments; ref. 35). Details on calendar epochs are provided in Supplemental Methods and Supplemental Table 9. Covariable imbalance before and after matching was evaluated using the $L_{1}$ statistic. Matching for all analyses was excellent, with all postmatching $L_{1} \approx 0$. Postmatch data sets were generated specifically for each main and subgroup analysis as described in Results (Figure 1).

The WHO PS is a modified 6-point scale, adapted from the WHO R\&D Blueprint Group to assess disease severity and measure clinical improvement in hospitalized patients (Supplemental Table 10 and refs. 29, 36). Patients were assigned a daily WHO PS based on their most severe status that day.

Model fitting. All mortality analyses were examined for significant contributions of facility effect using a shared frailty model in comparison with a Cox proportional hazards regression model (37). Additionally, the assumption of proportional hazards of a Cox proportional hazards regression model was examined and, if violated, an AFT model was implemented to examine consistency of effects (38).

We conducted a generalized linear mixed model (GLMM) approach for longitudinal data to examine trends in the daily RTRM probabilities over length of stay (RTRM trajectories) as a surrogate of progression/recovery. GLMM allowed for nesting of longitudinal observations under each patient included in the data set. For RTRM trajectories, all nesting parameters were examined for significant contributions to the model. GLMM models included random intercepts for patient-level RTRM trajectories and facility nesting.

Subgroup analyses. Additional analyses were performed to examine the association of CP with mortality depending on a patient's disease severity. Intubation is representative of severe disease. To perform subgroup analyses on intubated versus nonintubated patients, the cohorts were rematched to exclude intubation as a matching variable so that the effect of intubation could be evaluated (Figure 1). Patients were considered intubated if they had a record of intubation prior to or on the day of transfusion or pseudo-baseline for the intubation subgroup analysis, but those intubated within the first 48 hours of admission were excluded. Finally, we conducted exploratory analyses examining the association of days from admission to transfusion with mortality specifically within the CP cohort. The initial model examined the days from admission to transfusion as a continuous variable in relationship with mortality outcomes. Additionally, we stratified the main RTRM sample into 2 different groups, 0 to 3 and 4 to 7 days, to examine the association between CP and mortality within a given transfusion window. We rematched each of these cohort transfusion windows separately on baseline RTRM score, calendar epoch, intubation during hospitalization (excluding intubations in first 48 hours after admission), sex, age, and race/ethnicity.

General considerations. All analyses were performed using R, version 3.6.3, with the following packages: cem, survival, coxme, and lme 4 (39). The pseudo-baseline assignment was conducted using an automated macro in SAS 9.4 (SAS Inc.; ref. 40). An $\alpha$ level of less than or equal to 0.05 was considered significant for all models and analyses.

\section{Study approval}

This study was supported by HCA Healthcare and conducted in accordance with US regulations, applicable ICH E6 international standards of good clinical practice, and institutional research policies and procedures. This research was performed under a master retrospective protocol (MR 01) approved under expedited review by an external governing institutional review board (IntegReview/Advarra) and granted a waiver of informed consent.

\section{Author contributions}

SAAE oversaw all aspects of the study, including conceptualizing the study design and directing its implementation, and also contributed to the development of the statistical analysis plan, interpretation of results, and manuscript writing and review. AJ contributed to the study design, developed the statistical analysis plan, performed all data cleanup and data set construction, coded and implemented all analyses, interpreted results, and contributed to manuscript writing and review. SAAE is listed as the first co-author as she served as the research investigator leading the data science and data management groups, which AJ managed. JSAR and MW provided clinical insight and contributed to results interpretation and manuscript preparation and review. CM contributed to EHR data curation and sourcing, data verification, and manuscript review and editing. PD performed preliminary data cleanup, analyses, pseudo-baseline assignments, and manuscript review and editing. DS contributed to RTRM modeling, results interpretation, and manuscript preparation. LES provided oversight and contributed to overall study design, results interpretation, and manuscript review. MC contributed to resource allocation, results interpretation, and manuscript review. GF provided resource allocation and general oversight and contributed to results interpretation and manuscript review. HAB provided resource allocation and general oversight and contributed to results interpretation and manuscript review. CFL contributed to study conception and design, provided clinical insight, and contributed to results interpretation and manuscript preparation and review.

\section{Acknowledgments}

The authors wish to acknowledge Faisal Cheema, who contributed to operationalizing our HCA EAP CP program and provided clinical insight. Susan Garwood also provided clinical insight. We 
would also like to thank Troy Gifford, Ryan Patrick, Chris Guthrie, Cecile Roman, Arielle Fisher, Jessica Correia, Daniel Luckett, Kathryn Hopkins-McGill, and Shaita Picard for their contributions to electronic data extraction, management, and curation for the HCA Healthcare COVID-19 registry. Thank you to Molly Altman and Andrew Stillwell for providing project management support. We would also like to acknowledge Mandelin Cooper, Mitch Davis, and Laura McLean in the Clinical Operations Group for their parallel COVID-19 work that provided insight into HCA Healthcare treatment recommendations for the date epochs as well as variable sourcing and mapping suggestions. Many thanks to Sybil Hyatt, Sally Mathews, and the HRI Research Operations team as well as Michael Frost and the HRI Data Operations Team for their manual chart review efforts in confirming COVID-19 cases and identifying CP cases early in the pandemic for the HCA Healthcare COVID-19 Registry. We are grateful for our Forward Pathology Solutions and Metro Infectious Disease Consultants colleagues in Kansas City, Missouri, USA, for their continued support of JSAR and MW collaborations. We would also like to acknowledge our blood-bank collaborators who provided insight and serology on donor plasma, including Pampee Young at the American Red Cross and Larry Dumont at Vitalant, as well as our colleagues at Versiti. Finally, we would like to thank our collaborators at Mayo Clinic and Michigan State University, Rickey Carter and Nigel Paneth, for their post hoc discussion of the findings in context with current methodologies and CP literature. The views expressed in this publication represent those of the author(s) and do not necessarily represent the official views of HCA Healthcare or any of its affiliated entities.

Address correspondence to: Charles F. LeMaistre, Physician in Chief for Hematology, Sarah Cannon, 1100 Dr. Martin L. King Jr. Blvd., Suite 800, Nashville, Tennessee 37203, USA. Phone: 1.615.524.4105; Email: Charles.LeMaistre@SarahCannon.com.
1. Abolghasemi H, et al. Clinical efficacy of convalescent plasma for treatment of COVID-19 infections: results of a multicenter clinical study. Transfus Apher Sci. 2020;59(5):102875.

2. Allahyari A, et al. Efficacy and safety of convalescent plasma therapy in severe COVID-19 patients with acute respiratory distress syndrome. Int Immunopharmacol. 2021;93:107239.

3. Altuntas F, et al. Convalescent plasma therapy in patients with COVID-19. Transfus Apher Sci. 2021;60(1):102955.

4. Chai KL, et al. Convalescent plasma or hyperimmune immunoglobulin for people with COVID19: a living systematic review. Cochrane Database Syst Rev. 2020;10:CD013600.

5. Chowdhury FR, et al. Convalescent plasma transfusion therapy in severe COVID-19 patients- a safety, efficacy and dose response study: a structured summary of a study protocol of a phase II randomized controlled trial. Trials. 2020;21(1):883.

6. Janiaud P, et al. Association of convalescent plasma treatment with clinical outcomes in patients with COVID-19: a systematic review and meta-analysis. JAMA. 2021;325(12):1185-1195.

7. Jiang $\mathrm{W}$, et al. Clinical efficacy of convalescent plasma therapy on treating COVID-19 patients: evidence from matched study and a meta-analysis. Clin Transl Med. 2020;10(8):e259.

8. Joyner MJ, et al. Convalescent plasma antibody levels and the risk of death from Covid-19. N Engl JMed. 2021;384(11):1015-1027.

9. Liu STH, et al. Convalescent plasma treatment of severe COVID-19: a propensity score-matched control study. Nat Med. 2020;26(11):1708-1713.

10. Piechotta V, et al. Convalescent plasma or hyperimmune immunoglobulin for people with COVID-19: a living systematic review. Cochrane Database Syst Rev. 2020;7:CD013600.

11. Ripoll JG, et al. Convalescent plasma for infectious diseases: historical framework and use in COVID19. Clin Microbiol Newsl. 2021;43(4):23-32.

12. Rogers R, et al. Convalescent plasma for patients with severe coronavirus dDisease 2019 (COVID19): a matched cohort study. Clin Infect Dis. 2021;73(1):e208-e214.
13. Salazar E, et al. Treatment of coronavirus disease 2019 patients with convalescent plasma reveals a signal of significantly decreased mortality. Am J Pathol. 2020;190(11):2290-2303.

14. Shenoy AG, et al. Early mortality benefit with COVID-19 convalescent plasma: a matched control study. Br J Haematol. 2021;192(4):706-713.

15. Tworek A, et al. Convalescent plasma treatment is associated with lower mortality and better outcomes in high-risk COVID-19 patients - propensity-score matched case-control study. Int J Infect Dis. 2021;105:209-215.

16. United States Food and Drug Administration (FDA). Recommendations for Investigational COVID-19 Convalescent Plasma. https:// www.fda.gov/vaccines-blood-biologics/ investigational-new-drug-ind-or-deviceexemption-ide-process-cber/recommendationsinvestigational-covid-19-convalescentplasma\#Pathways\%20for. Updated February 11, 2021. Accessed August 31, 2021.

17. Mayo Clinic. Exanded Access Program (EAP) for Convalescent Plasma. Accessed September 15, 2021. https://www.uscovidplasma.org/

18. AlQahtani M, et al. Randomized controlled trial of convalescent plasma therapy against standard therapy in patients with severe COVID-19 disease. Sci Rep. 2021;11(1):9927.

19. Bandopadhyay P, et al. Nature and dimensions of the systemic hyper-inflammation and its attenuation by convalescent plasma in severe COVID-19 [published online January 12, 2021]. JInfect Dis. https://doi.org/10.1093/infdis/jiab010.

20. Recovery Collaborative Group. Convalescent plasma in patients admitted to hospital with COVID-19 (RECOVERY): a randomised controlled, open-label, platform trial. Lancet. 2021;397(10289):2049-2059.

21. Sarkar S, et al. Convalescent plasma is a clutch at straws in COVID-19 management! A systematic review and meta-analysis. JMed Virol. 2021;93(2):1111-1118.

22. Self $W H$, et al. Passive immunity trial for our nation (PassITON): study protocol for a randomized placebo-control clinical trial evaluating COVID-19 convalescent plasma in hospitalized adults. Trials. 2021;22(1):221.

23. Simonovich VA, et al. A randomized trial of convalescent plasma in Covid-19 severe pneumonia. N Engl JMed. 2021;384(7):619-629.

24. Wood EM, et al. How should we use convalescent plasma therapies for the management of COVID19? Blood. 2021;137(12):1573-1581.

25. Klassen SA, et al. Convalescent plasma therapy for COVID-19: a graphical mosaic of the worldwide evidence. Front Med (Lausanne). 2021;8:684151.

26. O'Donnell MR, et al. A randomized double-blind controlled trial of convalescent plasma in adults with severe COVID-19. JClin Invest. 2021;131(13):e150646.

27. Thompson MA, et al. Association of convalescent plasma therapy with survival in patients with hematologic cancers and COVID-19. JAMA Oncol. 2021;7(8):1167-1175.

28. Kunze KL, et al. Mortality in individuals treated with COVID-19 convalescent plasma varies with the geographic provenance of donors. Nat Commun. 2021;12(1):4864.

29. Schlauch D, et al. Development of a real-time risk model (RTRM) for predicting in-hospital COVID-19 mortality [preprint]. https://doi.org/ 10.1101/2021.04.26.21256138. Posted on medRxiv April 28, 2021.

30. Casadevall A, et al. Convalescent plasma use in the United States was inversely correlated with COVID-19 mortality: did plasma hesitancy cost lives [preprint]? https://doi.org/10.1101/2021.04 .07.21255089. Posted on medRxiv April 16, 2021.

31. Goodhue Meyer E, et al. Selecting COVID-19 convalescent plasma for neutralizing antibody potency using a high-capacity SARS-CoV-2 antibody assay. Transfusion. 2021;61(4):1160-1170.

32. Dodd RY, et al. Change in donor characteristics and antibodies to SARS-CoV-2 in donated blood in the US, June-August 2020. JAMA. 2020;324(16):1677-1679.

33. Sethuraman $\mathrm{N}$, et al. Interpreting diagnostic tests for SARS-CoV-2. JAMA. 2020;323(22):2249-2251.

34. Iacus SM, King G, and Porro G. Causal inference without balance checking: coarsened exact matching. Political Analysis. 2012;20(1):1-24. 
35. Yoon HA, et al. Treatment of severe COVID-19 with convalescent plasma in Bronx, NYC. JCI Insight. 2021;6(4):e142270.

36. World Health Organization (WHO) Working Group on the Clinical Characterisation Management of COVID-19 infection. A minimal common outcome measure set for COVID-19 clinical research. Lancet Infect Dis. 2020;20(8):e192-e197.
37. Bramante CT, et al. Metformin and risk of mortality in patients hospitalised with COVID-19: a retrospective cohort analysis. Lancet Healthy Longev. 2021;2(1):e34-e41.

38. Qi J. Comparison of Proportional Hazards and Accelerated Failure Time Models. Graduate thesis. University of Sasketchewan; 2009.

39. $R$ : A language and environment for statistical computing. Version 4.1.1. R Foundation; 2021. Accessed
August 31, 2021. https://www.R-project.org/. 40. Harvey R, et al. Random assignment of proxy event dates to unexposed individuals in observational studies: an automated technique using SAS. Paper presented at: Midwest SAS Users Group (MSUG) 2012; September 16-18, 2012; Minneapolis, Minnesota, USA. https://www. mwsug.org/proceedings/2012/PH/MWSUG2012-PH02.pdf. Accessed August 31, 2021. 